\title{
Structure of Colloidal Flocs in relation to the Dynamic Properties of Unstable Suspension
}

\author{
Yasuhisa Adachi, Azusa Kobayashi, and Motoyoshi Kobayashi \\ Graduate School of Life and Environmental Science, University of Tsukuba, Tsukuba, Ibaraki 305-8572, Japan \\ Correspondence should be addressed to Yasuhisa Adachi, yas@sakura.cc.tsukuba.ac.jp
}

Received 15 August 2011; Accepted 29 September 2011

Academic Editor: Eri Yoshida

Copyright (C) 2012 Yasuhisa Adachi et al. This is an open access article distributed under the Creative Commons Attribution License, which permits unrestricted use, distribution, and reproduction in any medium, provided the original work is properly cited.

\begin{abstract}
Dynamic behaviors of unstable colloidal dispersions are reviewed in terms of floc formation. Geometrical structure of flocs in terms of chemical conditions and formation mechanics is a key to predict macroscopic transportation properties. The rate of sedimentation and rheological properties can be described with the help of fractal dimension $(D)$ that is the function of the number of contacts between clusters $\left(N_{c}\right)$. It is also well known that the application of water soluble polymers and polyelectrolytes, which are usually used as a conditioner or flocculants in colloidal dispersions, critically affects the process of flocculation. The resulted floc structure is also influenced by the application of polymer. In order to reveal the roles of the polymers, the elementary rate process of polymer reaching to colloidal interface and subsequent reconformation process into more stable adsorption state are needed to be analyzed. The properties of permeable flocs and adsorbed polymer (polyelectrolyte) layers formed on the colloidal surfaces remain to be worked out in relation to inhomogeneous porous structure and electrokinetics in the future.
\end{abstract}

\section{Introduction}

Owing to the increasing social interest in environmental issues, interfacial phenomena and the behavior of colloidal particles have attracted attention [1]. Numerous colloidal particles exist in soil, water, and air environments. These particles have a large specific surface area where various chemicals can be adsorbed. Even substances that do not dissolve in water, such as dioxin, can be adsorbed and concentrated on the surface of colloidal particles with hydrophobic nature. If the colloidal particles are allowed to move with the water flow, they can be regarded as carriers for insoluble substances. Hence, it is important to analyze transport properties of colloidal particles to predict and control the movement and the fate of chemical substances in the environment. The dynamic behavior of colloidal particles depends critically on the way of flocculation. Therefore, the relationship between the microscopic flocculation process of colloidal particles and the macroscopic dynamics of the resultant flocculated system has been regarded as one of the most fundamental issues when considering the state and dynamics of chemical substances in aquatic environment. Figure 1 shows the relationship between various factors which control the flocculation process and macroscopic properties associated with flocculation. As shown in this figure, transport characteristics such as the rate of separation and rheological properties of the colloidal dispersion are largely affected by the structure of flocs. Consequently, many problems related to the transportation of colloidal particles are addressed by controlling the geometrical structure of flocs through kinetics of flocculation. As will be discussed later, an application of water soluble polymers and polyelectrolytes with an affinity to colloidal surfaces will critically influence flocculation processes. However, this process is not fully understood. In the present article, we introduce our effort using monodispersed spherical colloid as model system to establish the scheme of the study of the dynamics of unstable suspension placing an emphasis on the formation of colloidal flocs. Even though natural colloid does not obviously satisfy this condition, our strategy turned out to be useful to introduce the concept of fractal, to analyze the system on the basis of rate theory and evaluation of the role of polymer flocculants. 


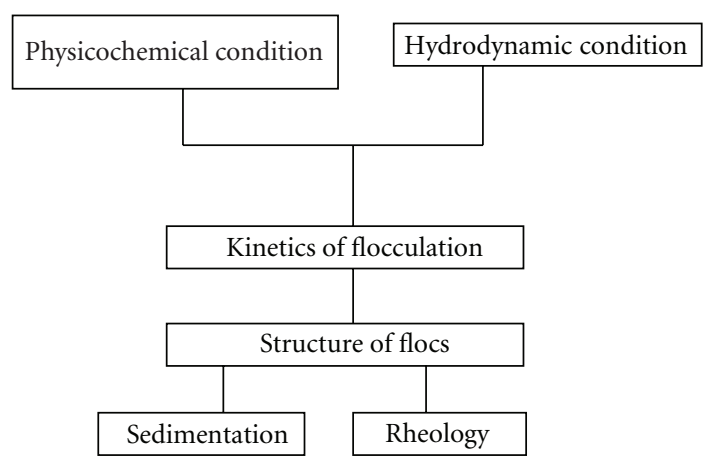

FIGURE 1: A correlation diagram of physical properties of a floc. (a)

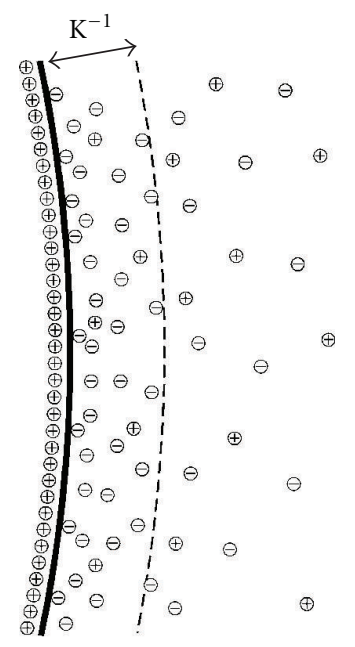

Diffusion of water molecules

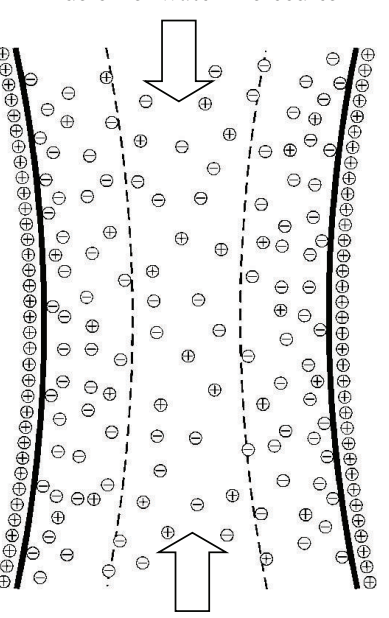

(b)
Figure 2: Schematic representations of a diffuse electrical double layer and the mechanism of generation of repulsive force by increasing the osmotic pressure. The chart was drawn on the basis of the ratio of the curvature radius of a colloidal particle to the Debye length $\kappa^{-1}$ to be 30 .

\section{Natural Flocculation and Behavior of Colloidal Particles}

Colloidal dispersions encountered in natural environments, such as suspension of clay minerals and organic substances in soil or turbid water, are usually thermodynamically unstable by themselves. If colloidal particles come close together, a universal van der Waals attractive force starts to interact between the particles. Because of this reason, when colloidal particles are brought close to each other by the effect of Brownian motion, fluid motion, and/or gravitational force, the colloidal particles start to flocculate and eventually form large flocs or aggregates.

The process of flocculation is usually analyzed by means of the rate theory. If all particle-particle collisions lead to flocculation, the flocculation rate is limited by particleparticle collisions; this type of flocculation is called rapid flocculation (or diffusion-limited aggregation). However, it is not always in reality, since a colloidal particle in water is

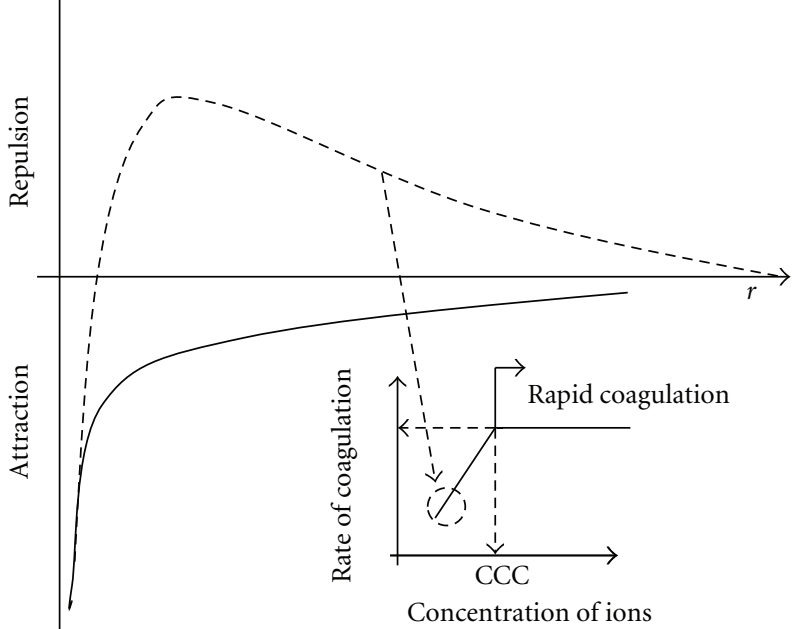

Figure 3: Potential between two particles and the coagulation velocity. When the salt concentration is low, the osmotic pressure results in the potential generation that causes positive repulsion (dotted line) with the expansion of a diffusion section of the electrical double layer. The coagulation velocity decreases because the particles overcome the potential.

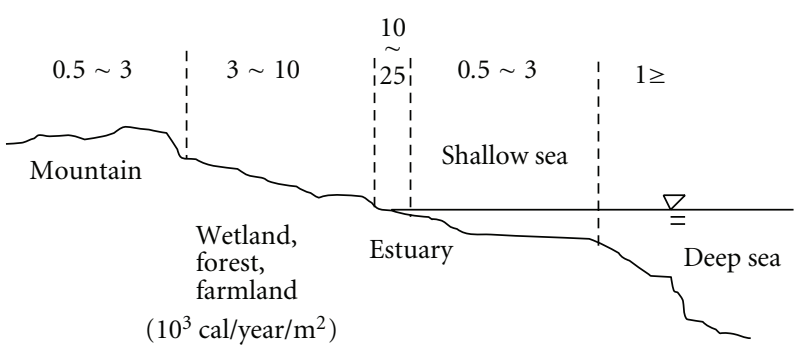

FIGURE 4: Biological primary production on the land and in the ocean.
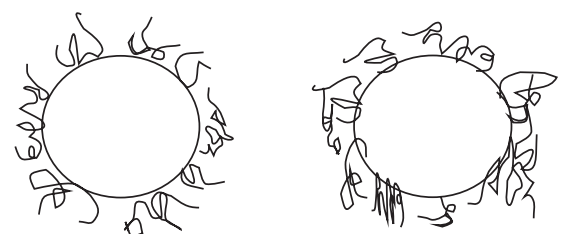

FIGURE 5: Coagulation inhibition by the steric hindrance of adsorptive polymers. When the polymer is adsorbed on colloidal particles, the protective effect hinders the particles from aggregation.

present with a considerable charge, thus attracting counter ions around the surface of the particles forming an ionic atmosphere. The development of the diffusive layer of ions will induce the increment in the osmotic pressure of the solvent in the gap region between the surfaces of two particles which are brought close together. Figure 2 shows a schematic representation of this situation. The development of the ionic diffusive layer prevents particles from adhesion. In other words, the flocculation of particles is inhibited by an energy barrier; hence, flocculation will take place at a significantly lower rate than that of rapid flocculation. This type of flocculation is called slow flocculation (or reaction-limited aggregation). Figure 3 shows schematically the relation between 


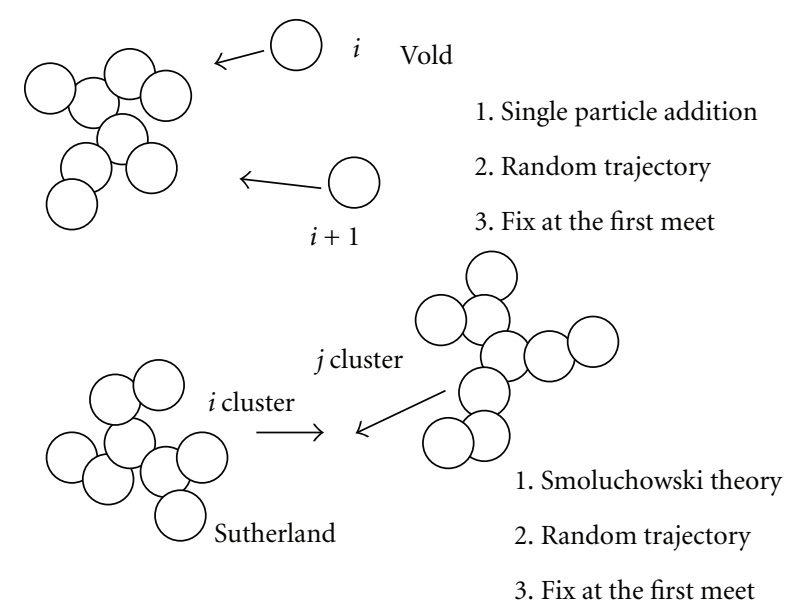

Figure 6: The single particle model (Vold) and the cluster-cluster addition model (Sutherlands).

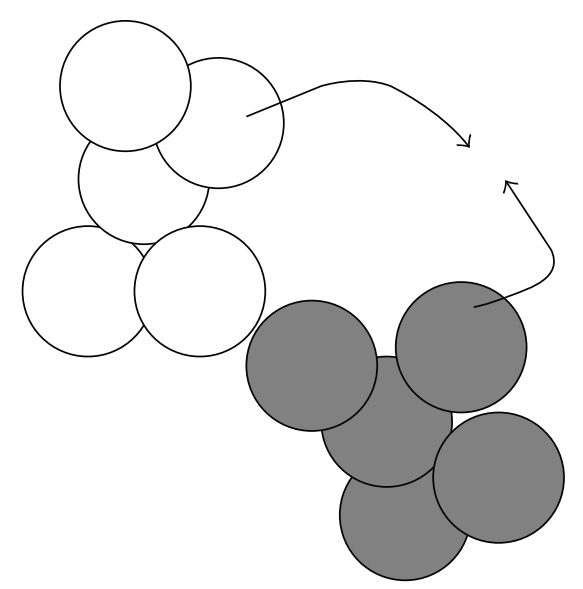

FIgURE 7: Rearrangement of collided clusters and increase in the number of their contacts.

the interacting potential and the rate of flocculation. In a low salt concentration, a positive potential that causes repulsion between particles is generated; hence, the rate of coagulation will become slow. The salt concentration corresponding to the point of inflection from slow to rapid flocculation is called the critical coagulation concentration (CCC).

In the case of slow coagulation, clear flocculation is not always induced. With a decrease of ionic strength, the tendency to remain cloudy will be increased. Even if the water in a freshwater area downstream near the river mouth is turbid, the transparency of water increases as it approaches to the marine environment. This is because the flocculation of colloidal particles or turbid components does not occur in water with a low salt concentration and the suspensions of small impalpable particles with a low sedimentation velocity can be maintained. On the other hand, the salt content of sea water is higher than CCC because of which the ionic diffusion layer gets compressed to induce rapid coagulation; thus, large flocs of the turbid components settle. Figure 4 depicts the process of biological primary production as summarized by Odum [2]; significantly high biological production is observed in a tidal flat in a coastal zone. The tidal ebb and flow causes the mixing of seawater and freshwater; the oxygen supplied by this mixture greatly contributes to the high biological production. In addition to this, aggregation of nutrient salts collected in flocs greatly contributes to a significant increase in the biological production capacity.

On the other hand, the seawater in contaminated areas such as Tokyo Bay does not always become transparent even when the salinity is high. This may be because organic matters adhere to the surfaces of the turbid colloidal particles. For instance, when organic macromolecules such as surfaceactive agents and proteins are adsorbed on the surfaces of colloidal particles, the thermal motions of their molecular chains, which have a fixed end and are projected like sea grass, prevent coagulation of the colloidal particles (Figure 5). This coagulation inhibition caused by the steric hindrance of the absorbed macromolecules is called the steric stabilization by absorbed polymer chains. Hunter et al. reported that water-soluble natural organic matters such as humic substances were adsorbed on colloidal particles such as clay minerals and metal hydroxides, thereby hindering the coagulation of the colloidal particles in the estuary $[3,4]$.

In the coagulation and settlement processes involved in the water treatment, the physicochemical conditions in the region containing colloidal particles are artificially modified to induce flocculation of the particles. Generally, in wastewater, the particle surfaces are contaminated. Hence, a flocculant is added in order to modify the particle surfaces and to increase/promote coagulation. A water-soluble polymer (or polyelectrolyte) is widely used as a flocculating agent to form large flocs of the colloidal particles. As will be discussed later, the details of the mechanism behind the formation of floc structures including the effect of the properties of a polymer flocculant [5] have not been fully understood.

\section{Fractal Structure of the Floc}

A floc of colloidal particles has a very high void ratio and an irregular shape. As a floc grows, its density decreases exponentially as a function of the floc diameter. The exponential reduction of the density reflects the fractal structure of the floc. The geometrical property of such flocs can be analyzed by methods such as the computer simulation by Monte Carlo method. In particular, since 1980s, a vast amount of data on the geometrical properties of flocs has been obtained with the development of the concept of fractals. However, the original model, other than the current fractal model, of the floc simulation was reported already in the late 1950s. Vold $[6,7]$ proposed a model involving a single particle addition in which individual spherical particles on a randomly selected straight line collide sequentially with the mother floc positioned at the origin, and a flocgrows when the particles are fixed at the point of the first contact with the mother floc. Flocs made of up to 60 particles were numerically reproduced. The flocs had random and irregular shapes. However, the exponential reduction of their densities with the growth of the floc could not be reproduced in their study. 


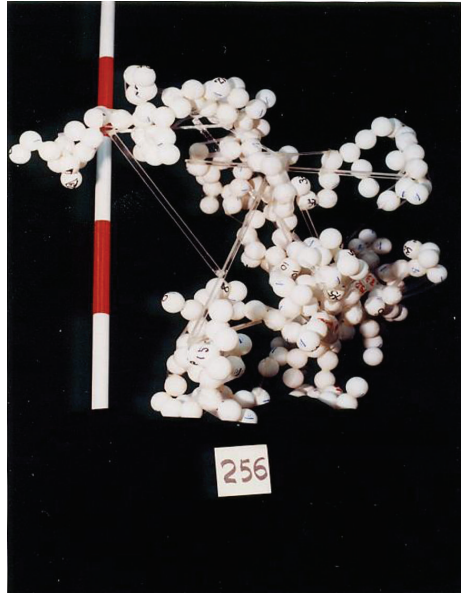

(a)

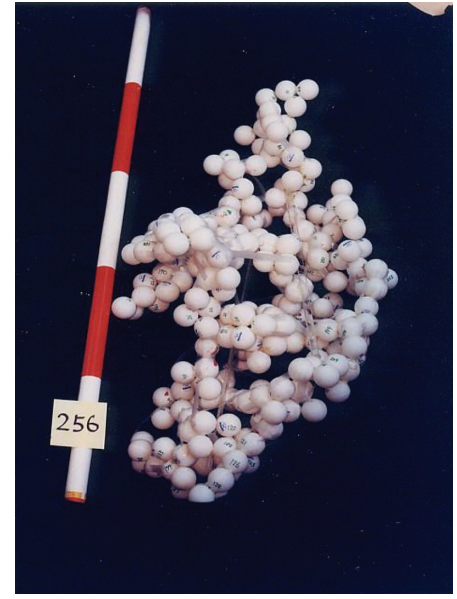

(b)

Figure 8: Floc model developed using ping-pong balls (number of particles: 256). (a) One cluster-to-cluster contact model. (b) Two clusterto-cluster contacts model [13].

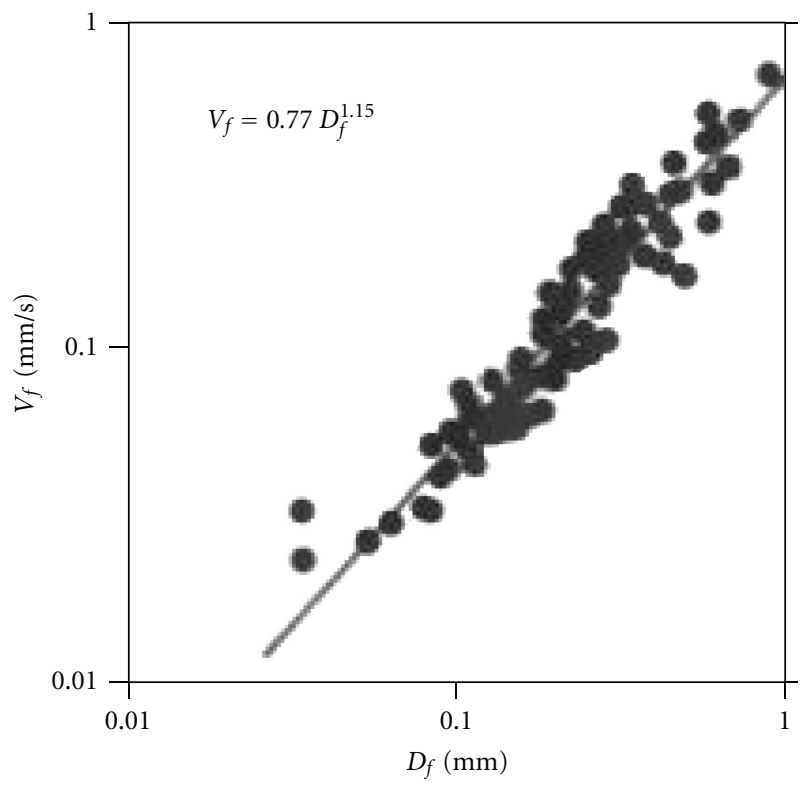

FIGURE 9: Relationship between the diameter and the settling velocity of kaolinite flocs [15].

Sutherland and Goodarz-Nia [8, 9] improved Vold's model and proposed a model of cluster-cluster aggregation by assuming that two clusters of colloidal particles collide repeatedly in sequence resulting in the growth of the floc; they proposed this model on the basis of computer simulations performed by applying the rate theory of coagulation developed by Smoluchowski [10], and they succeeded in reproducing the exponential reduction of the density with the floc growth (Figure 6). On the basis of the results obtained, the number of the colloidal particles in a floc is expressed in terms of a floc diameter function, as follows:

$$
i=\left(\frac{D_{f}}{d_{0}}\right)^{D},
$$

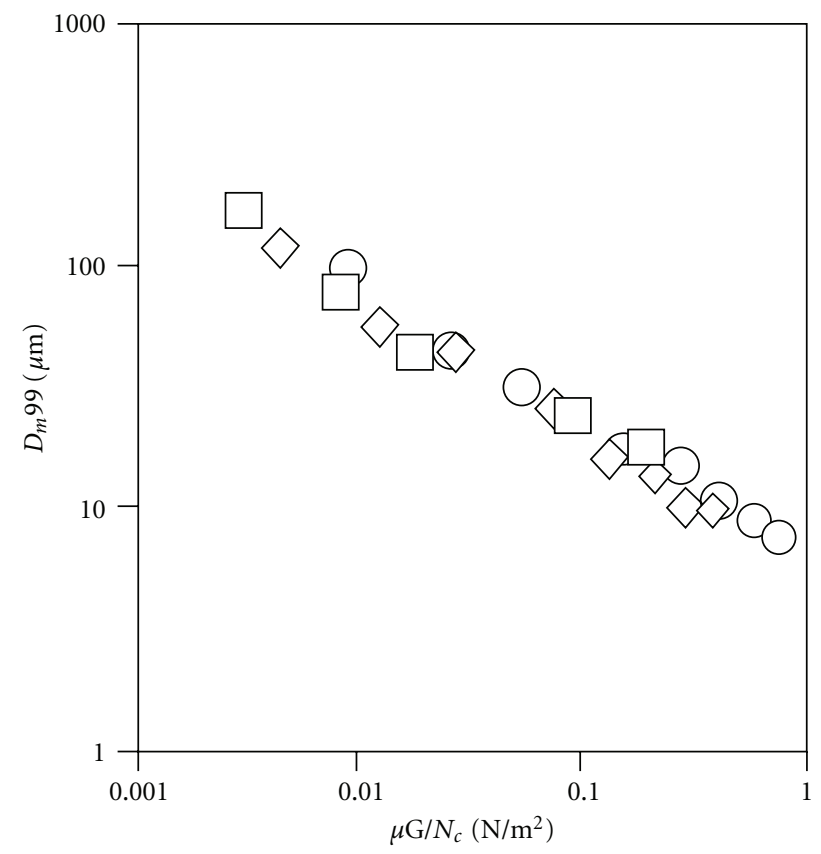

FIGURE 10: Relationship between the maximum diameter $\left(D_{m} 99\right)$ of the floc of polystyrene latex particles existing in the agitating tank and the strength of agitation. $G$ is the practical shearing velocity and $\mu$ is the viscosity coefficient. $\diamond ; N_{c}=1 ; \bigcirc: N_{c}=2 ; \square: N_{c}=3$ [17].

where $d_{0}$ is the diameter of primary particle and $D$ is the fractal dimension. From the simulation performed on the basis of Sutherland's model, $D$ is determined to be approximately 1.83; however, experimental results [11] are greater than this value and distributed. Considering that the difference between these values is a result of the way of formation of clusters, the authors proposed a two-point contact model. In this model, the clusters can roll and move to the next point of contact after a collision (Figure 7) $[12,13]$. Figure 8 shows the result of the simulation in which pingpong balls were considered. From the figure, it is apparent 


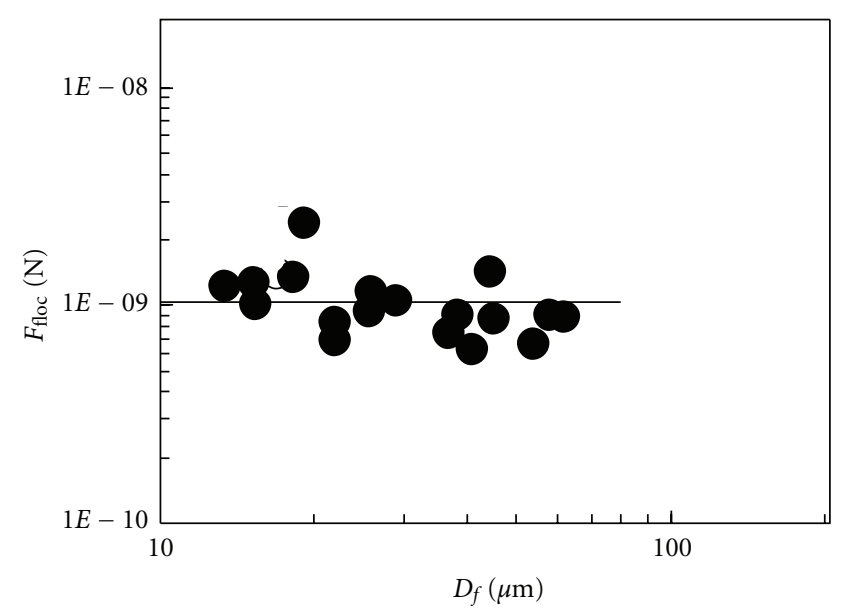

FIGURE 11: Cluster-to-cluster adhesive force calculated by a fracture experiment for contractile flow through the orifice. The experiment was conducted with a floc of polystyrene latex particles of the diameter of $2.8 \mu \mathrm{m}$.

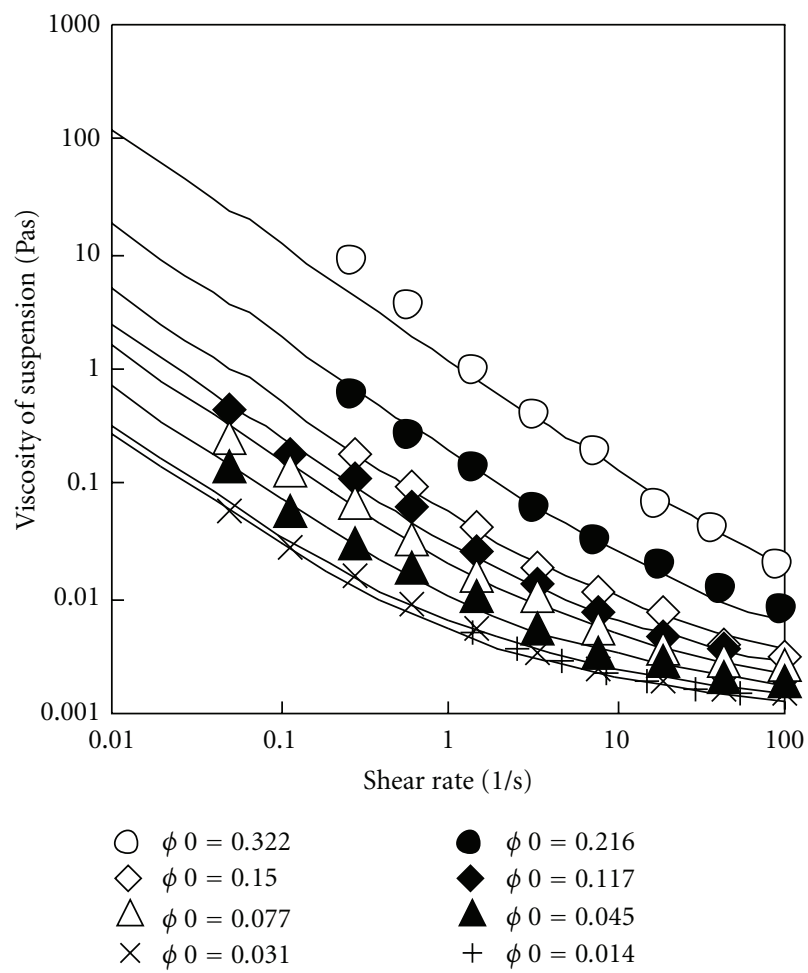

FIGURE 12: Analysis of the shear thinning viscosity of flocculated suspension of polystyrene latex with our model [19].

that by using the two-point contact model, a floc made of dense packing of particles can be obtained. Meakin and Jullien [14] performed the same simulation and confirmed that the fractal dimension increased to $2.13,2.18$, and 2.19 as the number of contacts between clusters $\left(N_{c}\right)$ was increased to 2 , 3 , and 4 , respectively.

\section{Fractal Structure and Physical Properties of Flocs}

The settling velocity of a single floc, $V_{f}$, is an indicator used to design a settling tank in the process of water and waste water treatment or to evaluate the transport diffusion of coagulated contaminants in the aquatic environments. $V_{f}$ can be obtained by analyzing the force balance involving terms for gravity, buoyancy, and the fluid resistance acting on a floc with fractal structure as follows [15]:

$$
V_{f}=\frac{g}{18 \alpha \mu}\left(\rho_{s}-\rho_{w}\right) \cdot d_{0}^{3-D} \cdot D_{f}^{D-1},
$$

where $\rho_{s}$ and $\rho_{w}$ are the densities of the colloidal particles and water (known), $\mu$ is the viscosity coefficient, and $g$ is the acceleration due to gravity, respectively. $\alpha$ is a coefficient reflecting the floc shape; for a spherical floc, the value of this coefficient is 1 . Figure 9 shows an example of experimental measurement of the settling velocity of flocs as a function of the floc diameter. On the basis of (2), the fractal dimension of the structure is determined from the slope of the plots presented in this figure.

As can be easily understood, the determination of the floc diameter at a given flow field is the most important issue to evaluate the rate of sedimentation. This is a so-called problem of floc strength and has been worked out for many years [16]. Kobayashi [17] proposed a simple model on the fracture strength of a floc that exists in a turbulent flow. That is, the floc will break up when the shear force $F_{\text {hyd }}$ of the fluid acting on the floc in the flow field exceeds the force retaining the floc $F_{\text {floc: }}$ :

$$
F_{\text {hyd }} \geq F_{\text {floc }} .
$$

The force retaining the floc is assumed to be the product of the adhesive force per contact between clusters $(f)$ and the number of cluster-to-cluster contacts $\left(N_{c}\right)$ :

$$
F_{\text {floc }}=f \cdot N_{c} .
$$

On the other hand, the shear force of the fluid acting on the floc is expressed by substituting the term for shear stress per unit area into the expression for the surface area of the floc:

$$
F_{\text {hyd }} \propto \mu G D_{f}^{2},
$$

where $\mu$ is the viscosity coefficient and $G$ is the substantive velocity gradient. By assuming (3) to be the condition for the fracture, $D_{f}$ is derived as follows:

$$
D_{f} \propto\left(\frac{N_{c}}{G}\right)^{0.5} .
$$

Figure 10 shows the size of the floc in a turbulent flow as a function of the fluid mixing intensity. The experimental result was plotted on a master curve by applying different $N_{c}$ values to flocs with different fractal dimensions. This result validates the concepts expressed in (4) and (5).

Equation (4) was validated by a floc fracture test by considering a contract flow through an orifice. The adhesive 


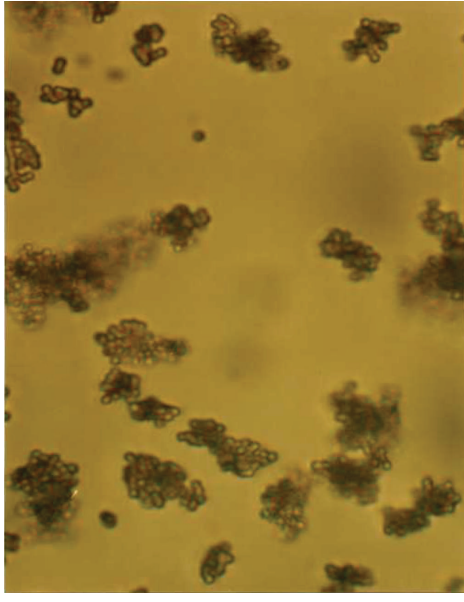

Slow coagulation in turbulent flow

(a)

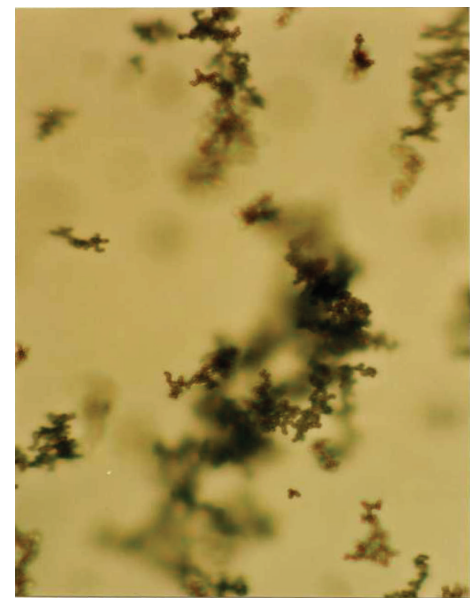

Rapid Brownian coagulation (KCI 1.19 M)

(b)

FIGURE 13: Flocs of polystyrene latex particles of the primary diameter $1.356 \mu \mathrm{m}$ formed in a turbulent flow (a) and flocs of the same particles formed by the rapid Brownian coagulation (b).

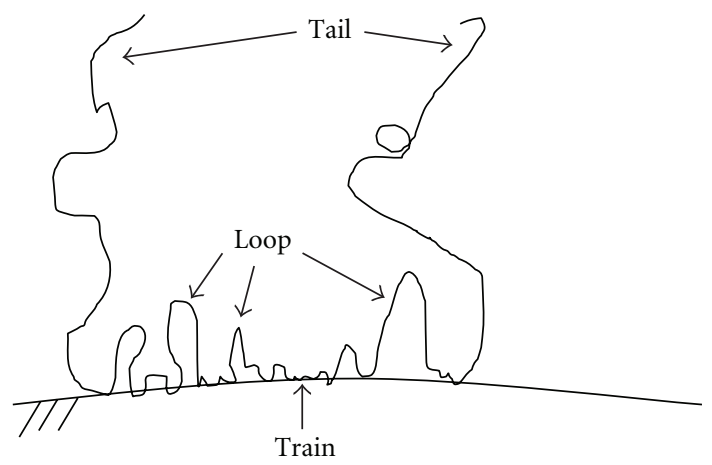

FIGURE 14: A typical conformation of a polymer chain adsorbed on the surface.

strength per contact within the floc was estimated to be of the same order as that of the adhesive force, including AFM measurements between surfaces (Figure 11) [18]. It is also interesting to note that (4) and (6) can be easily incorporated into a rheology model owing to simple forms of the terms used in these equations and can be used to describe non-Newtonian flows such as softening of materials (Figure 12) [19, 20].

\section{Rearrangement of Floc Structures}

As mentioned in the previous chapters, the number of contacts between clusters $\left(N_{c}\right)$ and the corresponding fractal dimension $(D)$ characterizing floc structures are of essential importance in settling/sedimentation and transport phenomena. However, physical mechanisms by which these factors are determined have not been fully understood as well as their contributions to floc formation. Meakin et al. $[14,21]$ ascribed the rearrangement of clusters to the condition of reaction-limited (or slow) coagulation, That is, there exists interparticle repulsion within a cluster, and demonstrated that the resulting flocs in this condition are more compact than those formed by diffusion-limited (or rapid) coagulation. On the other hand, we observed a cluster-to-cluster rearrangement when the initial particle concentration is high $[22,23]$ or the fluid flow is involved, even under the region of rapid coagulation (Figure 13) [24]. That is, interparticle repulsion is screened by the presence of concentrated salt ions. As indicated in Figure 13, the presence of the shear flow would induce rearrangement of the flocs leading to the formation of dense flocs.

\section{Formation of Flocs Enhanced by Polymers}

For polymer-induced flocculation, the presence of polymers additionally influences rearrangement and corresponding floc properties. In fact, water soluble polymers are often utilized as flocculants in water and waste water treatments to enhance the rate of flocculation and produce large, dense, and strong flocs to facilitate solid-liquid separation. When the flocs are large in size, having a nearly spherical, dense, and packed structure, they are referred to as pellet flocs. Napper [25] conducted experiments varying physicochemical conditions during flocculation operation and reported that pellet flocs are formed in the concentrated colloidal suspension only when a certain level of polymer is mixed under a proper stirring intensity. The fractal dimension of pellet flocs approaches to 3.0 since branching structures within a cluster are diminished. Formation of these pellet flocs provides obvious evidence for rearrangement of interacting clusters. It is noteworthy that they are formed only in the presence of shear flow. This observation leads us to a possible explanation that the contact points of the clusters are flexible due to the adsorbed polymers in between so that the clusters can rearrange themselves quite easily driven by the shear force.

Let us now summarize the process of floc flocculation enhanced by adsorbing polymers. Two flocculation mechanisms are well known: bridging and charge neutralization. 


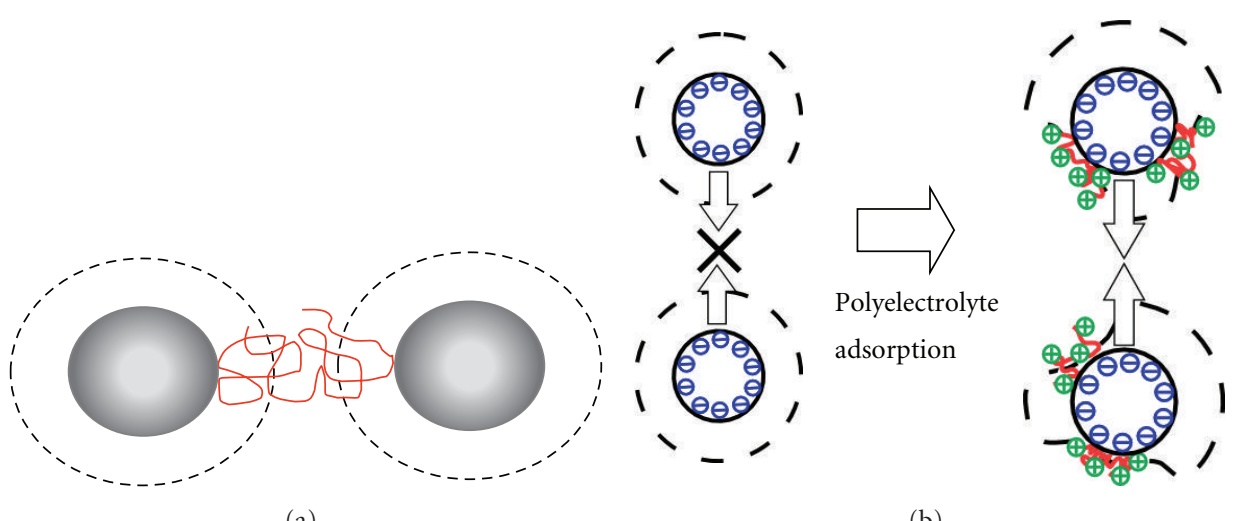

(a)

(b)

FIGURE 15: Flocculation mechanisms: (a) bridging and (b) charge neutralization. The dashed line represents the electric double layer.

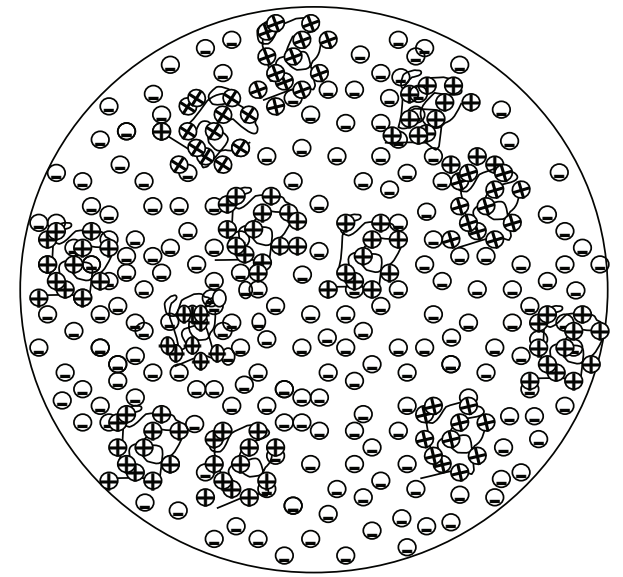

FIGURE 16: A mosaic-like distribution of highly charged polyelectrolytes on a particle of the oppositely charge.

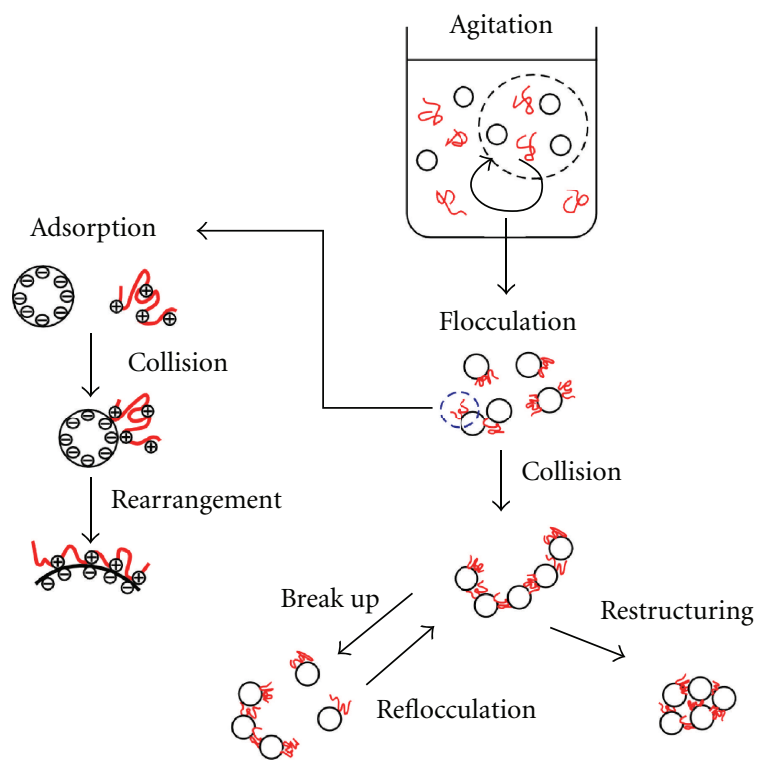

FIGURE 17: A schematic diagram of flocculation with adsorbing polymers [40].
Whether which mechanism is dominant is markedly dependent on the conformation of adsorbing polymers at colloidal surfaces. Once a polymer chain is adsorbed onto the surface of a particle, the attached chain undergoes a conformational change (referred to as reconformation) after a characteristic time toward a more stable configuration with loops, tails, and trains (Figure 14) [26]. If the adsorbing polymer has a high molecular weight, or the polymer chain is long, it is likely to result in the formation of protruding loops and tails extending into the solution side. These protruding segments on a particle can reach to other particles and adsorb thereon, thus bridging the particles together as a floc as illustrated in Figure 15(a). Flocculation on this basis is referred to as bridging flocculation, which is firstly proposed by Smellie Jr. and La Mer in 1958 [27]. For bridging there must remain an unoccupied portion on the surface where polymer chains adsorbed on another particle can attach to, otherwise the particles will be stabilized and flocculation will not take place. A very first work on the efficiency of bridging flocculation with an account of the surface coverage was proposed by Fleer and Lyklema [28]. According to their concept, the probability of bridge formation is proportional to the fractional surface coverage $\theta$ and the uncovered fraction $(1-\theta)$. Although this model accounts for the experimental observation that the formation of bridges is more facilitated when the surface coverage is low, a quantitative estimation based on this idea is hardly achieved since numerous kinetic factors regarding adsorption are involved as well as complex structures of adsorbed polymers which make the measurement of surface coverage complex. The extending polymer chains must have a greater than twice the thickness of the electric double layer $\kappa^{-1}$ around a particle to form a bridge. That is, bridging flocculation can take place if the extent of the adsorbed polymer layer $\delta$ is greater than $2 / \kappa$, while no bridging flocculation develops as $\delta<2 / \kappa$. This is confirmed experimentally, for instance, by Gregory [29].

Another mechanism of flocculation is called charge neutralization. Adsorption of a charged polymer onto the surface of an oppositely charged particle results in the reduction in the net charge of the particle. The neutralization of the surface charge lowers electrostatic repulsion acting between the particles, thus induces flocculation. Figure 15(b) 

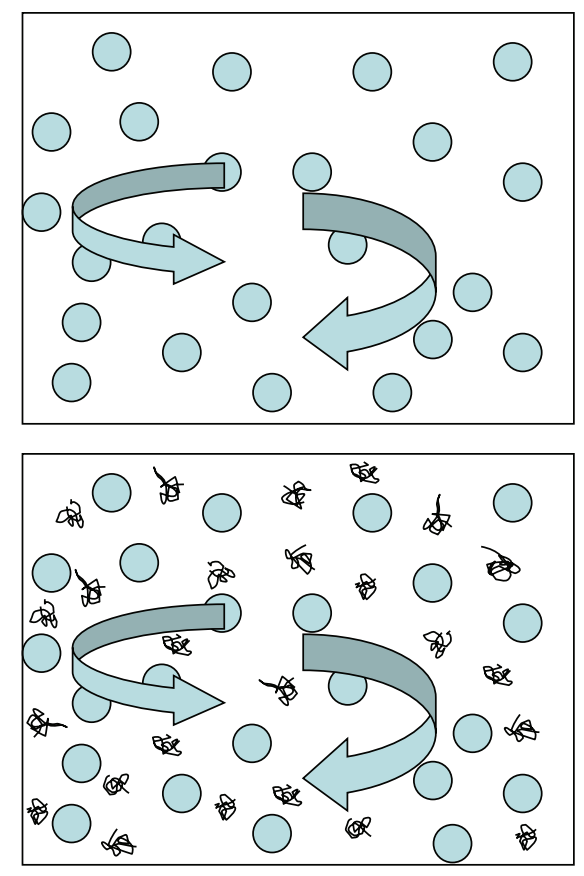

Evaluate the mixing flow in

terms of collision

(1) Collision $=$ rate of coagulation

$\frac{d N}{d t}=\alpha_{s} \frac{4}{3} \sqrt{\frac{2 \varepsilon}{15 \nu}} R^{3} N^{2}$

(2) Exp. with polymer

$R \longrightarrow\left(R+\delta_{H}\right)$

Effect of polymer = (2) - (1)

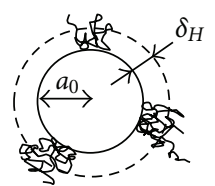

FIGURE 18: Normalization of mixing condition applied to analyze the influence of polymer addition.
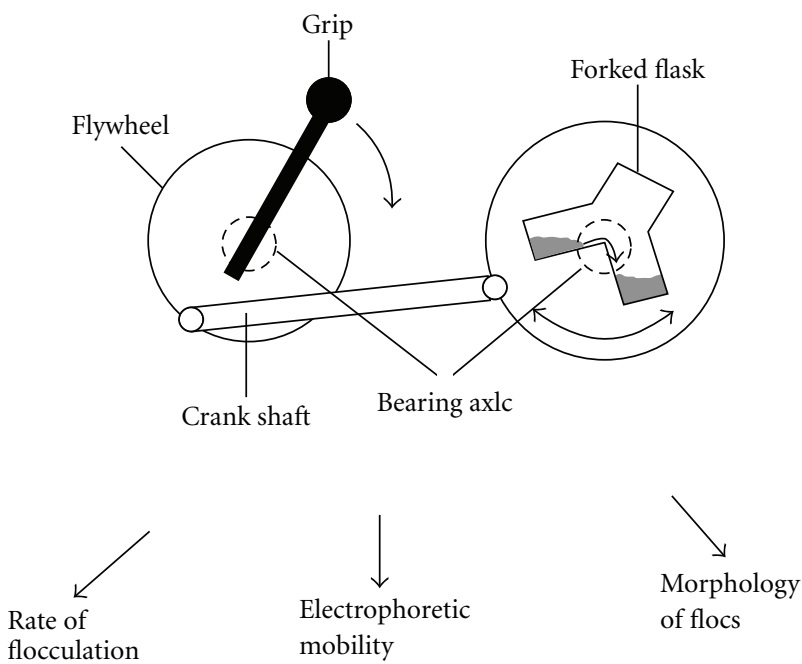

FIGURE 19: The end-over-end rotation apparatus to generate standardized mixing with a small volume of samples. After the mixing of a predetermined period, samples are taken up to be monitored by the rate of flocculation, the electrophoretic mobility and the morphology of flocs.

shows a schematic representation of this mechanism. One can imagine that the charged surface may not be completely covered with adsorbed polyelectrolytes even if the net electric potential of the particle is neutralized. In this case, mosaic-like distributed polyelectrolytes can interact with unoccupied surfaces of other particles to form a floc (Figure 16). The attraction in this manner is called electrostatic patch effects, which is proposed by Higashitani et al. [30] through a flocculation experiment of polystyrene particles with strongly charged cationic polyelectrolytes. Electrostatic interaction acting between a particle and polyelectrolyte is a function of the ionic strength of the solution as well as the charge density of polyelectrolyte; accordingly, the process of charge neutralization is affected by both parameters.

Each of the flocculation mechanisms described above is governed by the process of polymer adsorption and collision of colloidal particles, which markedly influence the conformation of the polymers at the surfaces. A schematic diagram of flocculation induced by polymer addition is shown in Figure 17. When polymers are added to the colloidal dispersion, the system is brought to the state far from the equilibrium. As seen in the diagram, the flocculation procedure consists of following several elementary processes under the mixing conditions, which usually is the turbulent flow:

(i) mixing of the polymeric flocculants into a homogeneous solution,

(ii) collision between colloidal particles,

(iii) transportation of a polymer chain toward the surface of a particle,

(iv) reconformation of an adsorbed polymer chain on the surface of a particle,

(v) formation of bonds (or bridges) between colloidal particles,

(vi) rearrangement and breakage of floc structures.

All these elementary processes occur simultaneously. That is, a cluster fixation would take place at the same time with or at the early stages of polymer adsorption and collision of the particles. Variations in floc structures produced in 


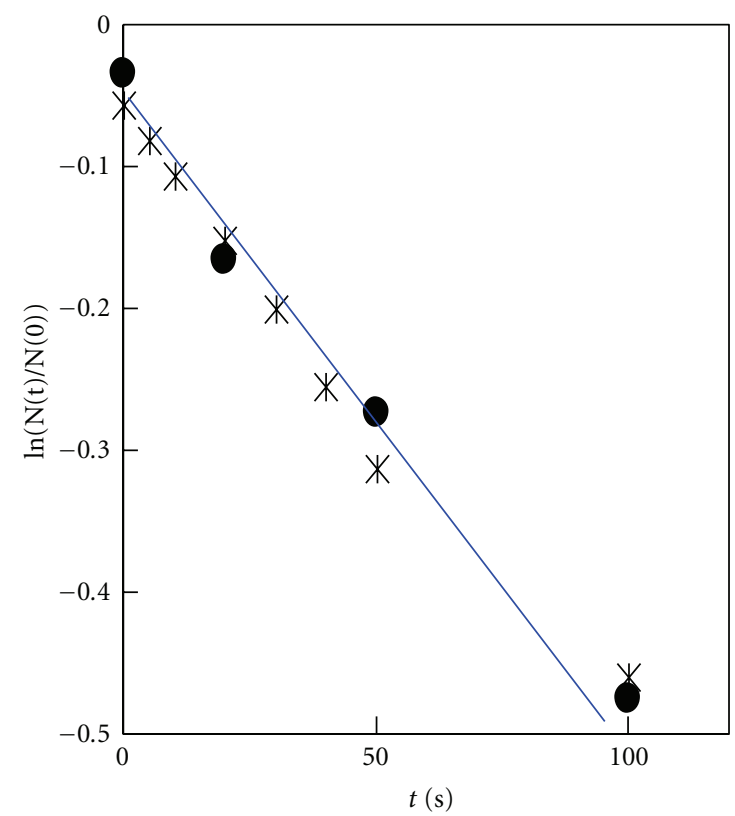

(a)

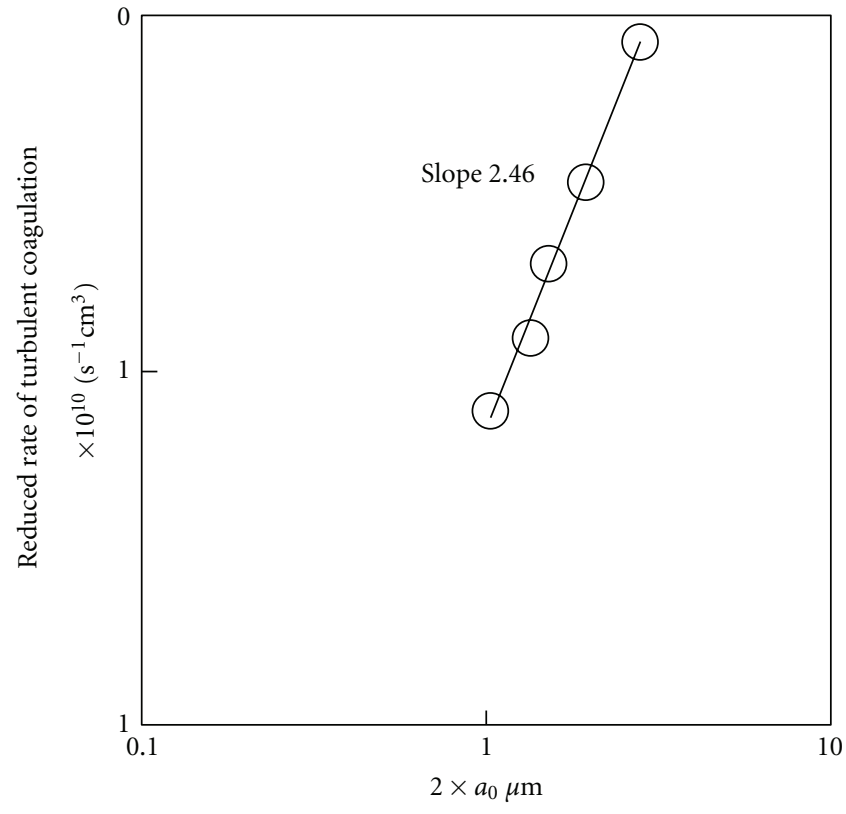

(b)

Figure 20: The result of the progress of salt-induced rapid coagulation in the standardized mixing. (a) Temporal evolution of the number concentration of flocs. (b) Reduced rate of coagulation as a function of the particle diameter.

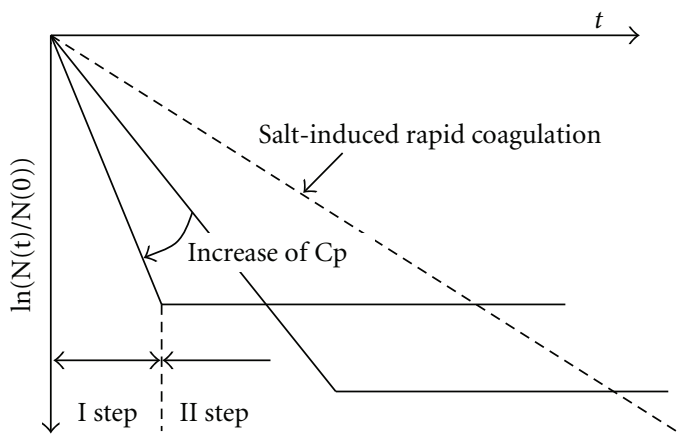

FIGURE 21: Concept of the progress of flocculation with addition of the excess amount of adsorbing polymers [47].

different physicochemical conditions support this idea [2325]. Therefore, the analysis of kinetic aspects listed previously is an essential requirement in order to elucidate the process of rearrangement of flocs structures.

\section{Monitoring Rearrangement of Floc Structures}

Although it is now obvious that the process of particle collision as well as polymer adsorption at early stages of flocculation has significant impacts on the floc formation, including cluster fixation and corresponding rearrangement of floc structures, few studies are available on the mechanism of action. In order to investigate dynamic aspects of colliding particles under the turbulent flow, the authors developed a reproducible method of colloidal mixing and applied this technique to assess nonequilibrium behavior of polymer adsorption (Figure 18). Assuming that all collisions between particles lead to coagulation, one can evaluate the turbulent flow of mixing by monitoring the rate of coagulation. First, let us consider a monodispersion of colloidal particles undergoing rapid coagulation with a sufficient content of salt. The rate of coagulation under the mixing flow was worked out by Adachi et al. [31]. Also, Saffman and Turner [32] confirmed the correlation of collision efficiently owning to hydrodynamic interaction derived by van de Ven and Mason [33] evoking the concept of the local isotropy of turbulence proposed by Komogrov. The progress of coagulation is expressed with a temporal variation of the total particle number concentration $N(t)$ as

$$
\frac{d N(t)}{d t}=-\frac{1}{2} \alpha_{T} \sqrt{\frac{8 \pi \varepsilon}{15 \nu}}\left(2 a_{0}\right)^{3} N(t)^{2},
$$

where $\varepsilon, v$, and $a_{0}$ are the rate of energy dissipation per unit mass of the fluid, kinematic viscosity, and the radius of a colloidal particle, respectively. $\alpha_{T}$ is the collision efficiency reflecting the hydrodynamic interaction. For rapid coagulation, an approximate expression for $\alpha_{T}$ is derived by substituting the effective shear rate of turbulent flow in the numerical calculation result of Adachi et al. [34] taking into account van der Waals attractive forces in the trajectory analysis of two colloidal particles in the laminar shear field. That is,

$$
\alpha_{T}=\left(\frac{A}{36 \pi \mu a^{3} 0 \sqrt{4 \varepsilon / 15 \pi \nu}}\right)^{0.18}
$$




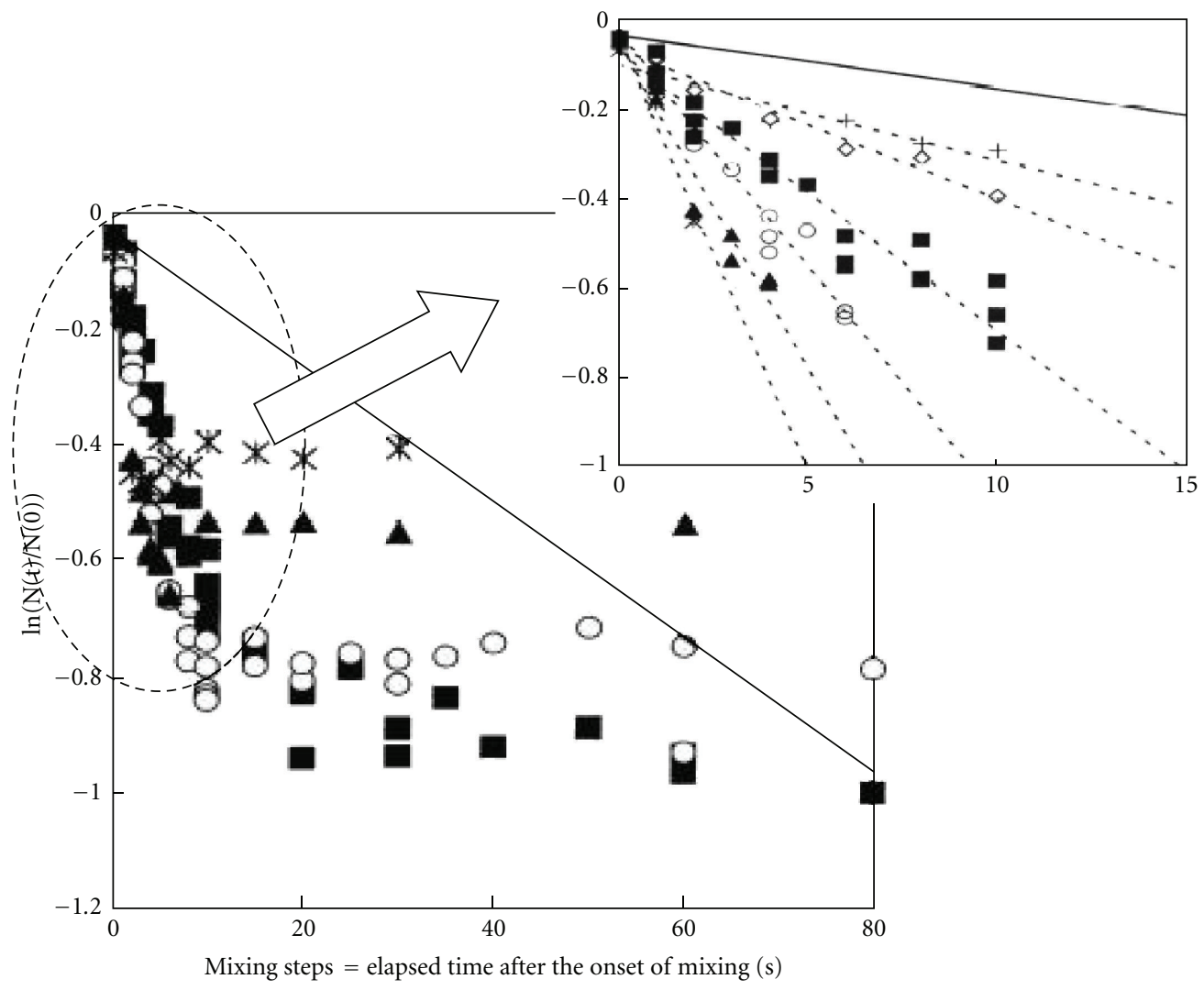

FIGURE 22: $\ln (N(t) / N(0))$ versus time for PEO-induced flocculation [47].

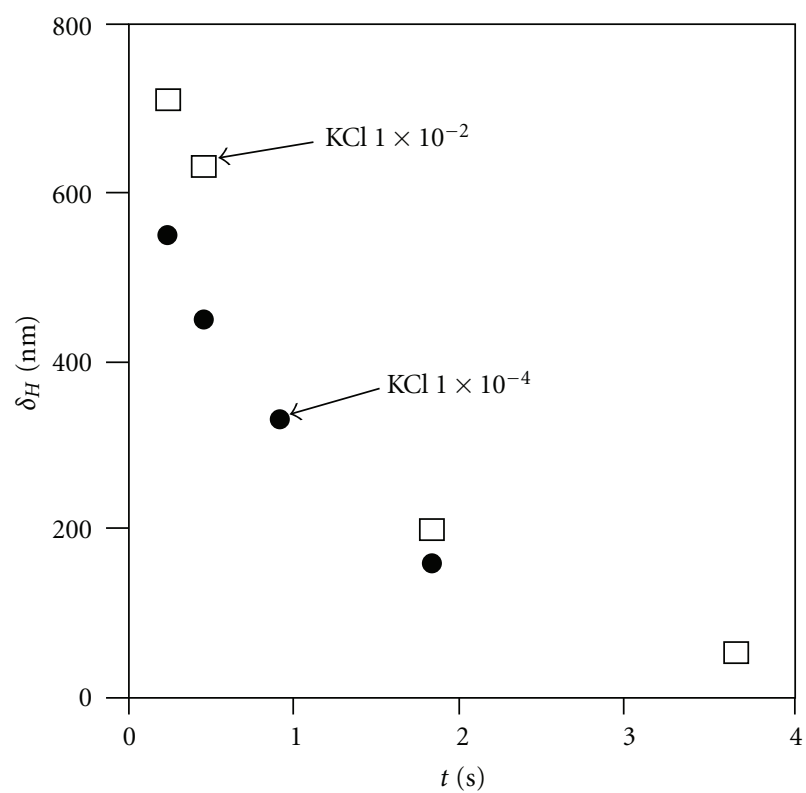

Figure 23: The thickness of a polymer layer $\delta_{H}$ as a function of the interval of polymer supply [47].

where $A$ is the Hamaker constant and $\mu$ is the viscosity. If the discussion is restricted to the early stage of coagulation, where the volume fraction of clusters can be assumed as

$$
\phi=N(t) \times \frac{4 \pi}{3} \times a_{0}^{3},
$$

then (7) yields the following approximate solution:

$$
\ln \frac{N(t)}{N(0)}=-\alpha_{T} \sqrt{\left(\frac{24 \varepsilon}{5 \pi \nu}\right)} \phi t .
$$

Evaluation of mixing flow is now possible by simply measuring the number concentration of coagulating colloidal particles as a function of elapsed time using the monodispersed colloidal particles under the condition of rapid coagulation. In our experiment, an end-over-end mixing device was developed to realize normalization of mixing flow (Figure 19). Coagulation was performed with this apparatus and the rate of coagulation, the electrophoretic mobility, and the morphology of the resultant complex were monitored at each step of mixing. A typical result of the evolution of coagulation is given in Figure 20.

Furthermore, the authors used the aforementioned technique to investigate the effect of polymers on the flocculation of the same system. The temporal evolution of flocculation is shown in Figure 21. Flocculation is enhanced immediately after the addition of polymers (stage I) until the surface of the particles reaches saturation (stage II). Since the flocculation rate is greatly dependent on the size of colloidal particles as seen in (7), the effective thickness of the adsorbed polymeric layer $\delta_{H}$ can be obtained from the ratio of the flocculation rate enhanced by polymers to that of without polymer addition, using

$$
\eta=\frac{\left(a_{0}+\delta_{H}\right)^{3}}{\alpha_{T} a_{0}^{3}} .
$$




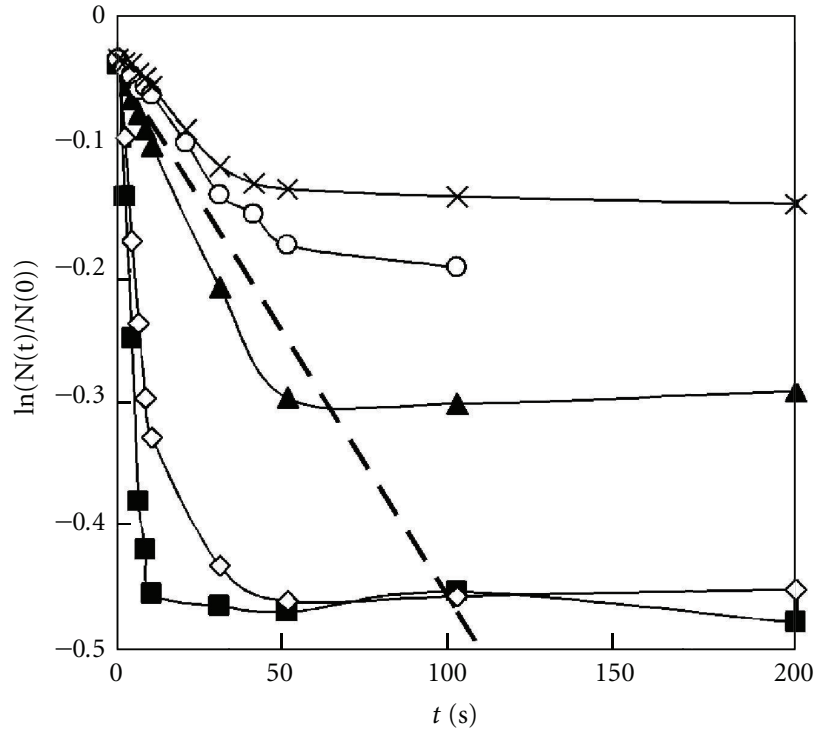

(a)

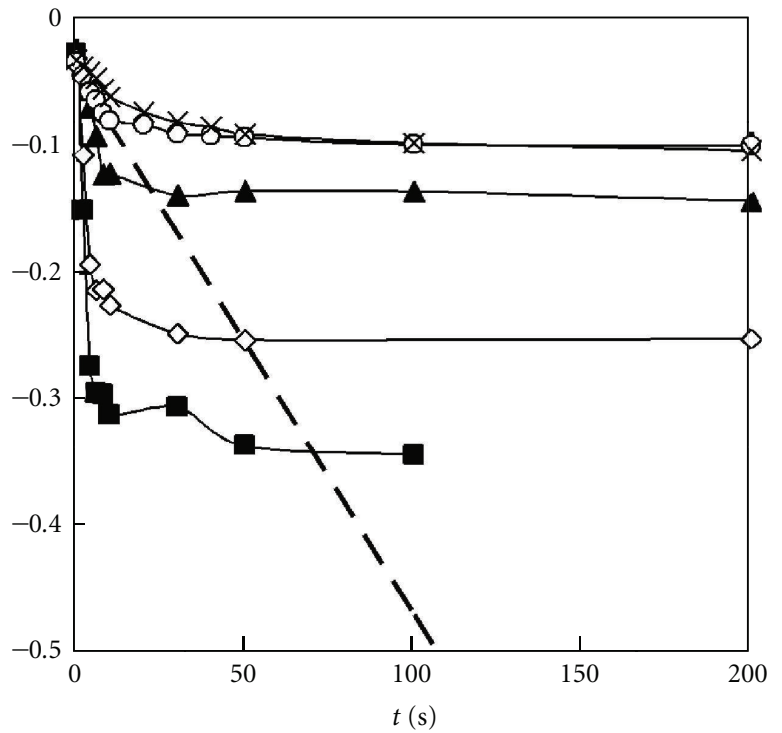

(b)

Figure 24: $\ln (N(t) / N(0))$ versus time for flocculation with polyelectrolytes of the concentration of $0.5 \mathrm{mg} / \mathrm{L} .(\mathrm{a}) \mathrm{KCl}=1.0 \times 10^{-2} \mathrm{M}$. (b) $\mathrm{KCl}=1.0 \times 10^{-4} \mathrm{M}$. The molecular weights of the polyelectrolytes are ( $\left.\mathbf{\square}\right) 4.9$ million, $(\diamond) 3.5$ million, $(\boldsymbol{\Delta}) 1.2 \mathrm{million}$, ( $\left.\bigcirc\right) 0.49$ million, and $(\times) 0.16$ million.

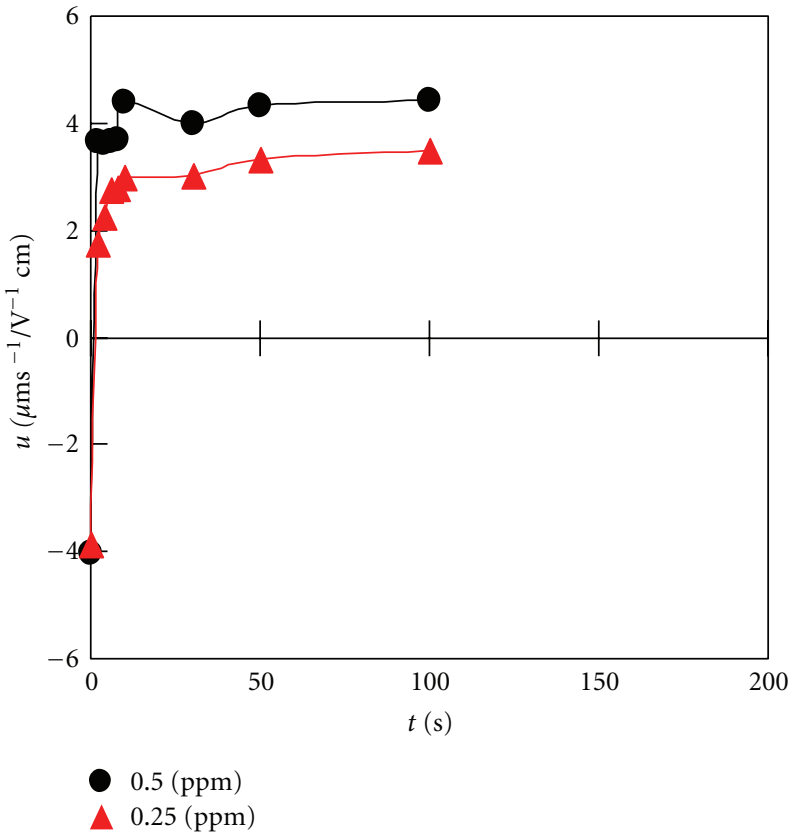

(a)

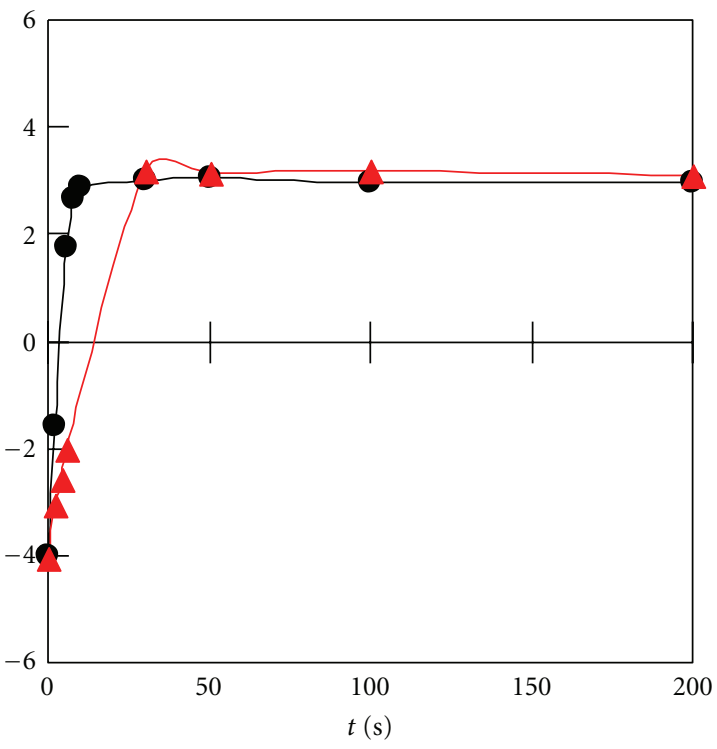

(b)

FIGURE 25: Electrophoretic mobility of the polyelectrolyte-PSL particle complexes versus the agitation time. $(\mathrm{a}) \mathrm{KCl}=1.0 \times 10^{-4} \mathrm{M}$. $(\mathrm{b})$ $\mathrm{KCl}=1.0 \times 10^{-2} \mathrm{M}$. The molecular weight of the polyelectrolyte was 4.9 million.

When the polymer concentration is high, it is likely that the particles carry freshly adsorbed polymers, and thus the effective collision radius is detected higher. Meanwhile, $\delta_{H}$ will be detected small if the polymer reconforms to a flattened structure on the surface when the polymer concentration is low. The time required for the saturation decreases with the polymer concentration, as expected. The flux of polymers toward a reference particle can be quantified by assuming a simple addition of the diffusion flux by Brownian motion and the collision induced by the turbulent flow, as

$$
J_{p}=4 \pi D_{O P} R_{O P} N_{p}+\sqrt{\frac{8 \pi \varepsilon}{15 \nu}}\left(a_{0}+a_{p}\right)^{3} N_{p}
$$

where $D_{O P}$ and $R_{O P}$ are the relative diffusion constant and collision radius of a polymer chain and a particle, 
respectively, and $a_{p}$ and $N_{p}$ are the radius and the number concentration of polymers, respectively. The authors conducted a flocculation experiment of polystyrene latex (PSL) particles induced by the excess addition of polyethylene oxide (PEO) of a nominal molecular weight of $5.0 \times 10^{6} \mathrm{~g} / \mathrm{mol}$ (Figure 22) [35]. Enhancement of the flocculation rate provides evidence for bridging flocculation. The initial rate of flocculation is greater for higher PEO concentration cases, indicating that $\delta_{H}$ for the collision radius grows with the PEO concentration. On the basis of (12) and defining the projected area of a single PEO chain as a square of its diameter, the relationship between $\delta_{H}$ and the interval of the polymer supply is clarified as shown in Figure 23. One can see that the reconformation of a polymer chain is disturbed when the surface is crowded with previously adsorbed polymers, that is, when the flux of polymers toward a particle is high. Note that the reconformation occurs within the order of a few seconds.

Most polymeric flocculants in practical applications are polyelectrolytes. They carry charged groups on their segments and thus have a strong adsorption affinity to the surface of oppositely charged particles. The temporal progress of flocculation of PSL particles induced by strong polyelectrolytes is shown in Figure 24 as a function of the molecular weight and ionic strengths [36]. The rate of flocculation is enhanced compared to that of salt-induced coagulation when the polymeric molecular weight is high because of formation of bridges; meanwhile the flocculation is not facilitated greatly when the shorter polyelectrolyte chains are used. Taking into account the neutralized net charge of the PSL-polyelectrolyte complex, one can reasonably estimate that charge neutralization was dominant in the latter case $[37,38]$. Interestingly, the electrophoretic mobility of PSL particle-polyelectrolyte complexes converges on the same plateau value regardless of the polymer concentration if the chain length is the same, except for the cases of high molecular weight chains under low ionic strength (Figure 25) [39]. Furthermore, the observation of the morphology of the complexes produced under the low ionic strength revealed that the rearrangement of particles is significantly hindered and the flocs are resulted in an open structure as shown in Figure 26. The explanation for the aforementioned results is given as follows. Under lower ionic strengths, polymer chains are rigidly adsorbed on the oppositely charged particles while they repel each other with strong electrostatic repulsion among charged segments, making both polymers and particles difficult to move as illustrated in Figure 27 [39-41]. In contrast, under the high ionic strength, that is, when polymer-particle electrostatic attraction and polymerpolymer repulsion are screened, adsorbed polyelectrolytes smoothly undergo reconformation and subsequently produced flocs easily rearrange their structures to have increased contact points. Interestingly, dependency of ease of rearrangement and structures of flocs on the ionic strength is reversed with and without polymer flocculants. The same trend is observed in a comparative study on floc structures of humic acids produced by various dual-coagulants of different electric properties [42]. Experimental results discussed previously imply the necessity of introducing a new concept

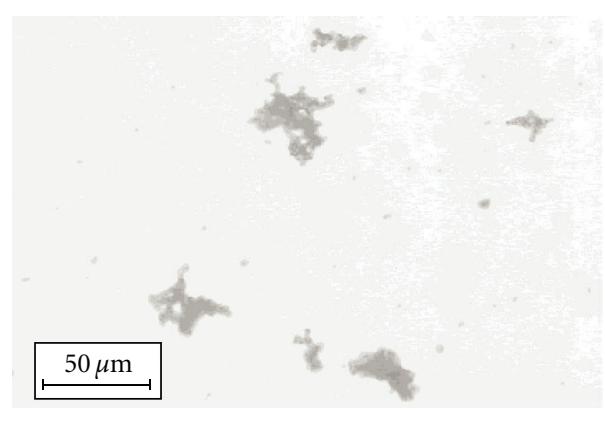

KCl: $1 \times 10^{-4}(\mathrm{~mol} / \mathrm{l})$

Polyelectrolyte Mw: $4.9 \times 10^{6}$

Concentration: $0.6(\mathrm{mg} / \mathrm{l})$

(a)

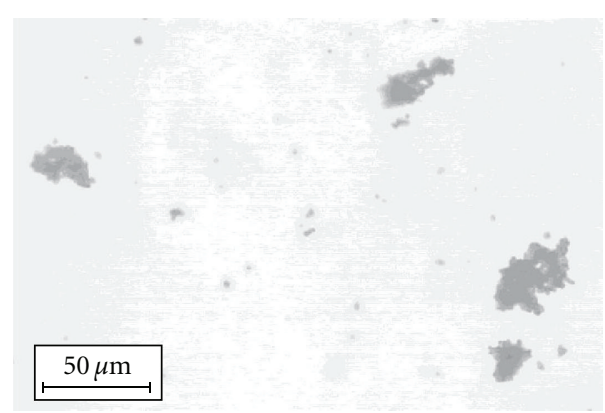

$\mathrm{KCl}: 1 \times 10^{-2}(\mathrm{~mol} / \mathrm{l})$

(b)

Figure 26: Microscopic photographs of polystyrene latex flocs formed by polyelectrolyte flocculants of a large molecular weight. When the ionic strength is low (a), low-density bulky flocs are formed. In contrast, high-density flocs are formed when the ionic strength is high (b) so that adsorbed polyelectrolytes undergo reconformation smoothly [39].

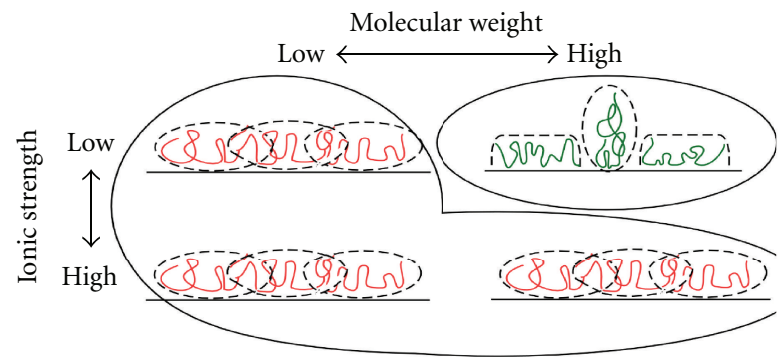

FIGURE 27: Polyelectrolytes adsorbed on the surface of a colloidal particle. The final pattern is affected by the adsorptive process when the ionic strength is low and the molecular weight is high.

on the way to fix contact points of clusters instead of a traditional idea of simple energy barrier.

\section{Future Perspectives}

We have discussed the importance of floc rearrangement in the earlier part of this review. Thus far, our discussion had been based on the fact that floc structures are determined by 
the process of coagulation/flocculation. Interestingly, some reports suggest that a further growth of flocs leads to the rise in the rate of coagulation $[43,44]$. That is, the floc structure itself has some influence on the progress of coagulation. Their interdependency remains to be elucidated. Also, in the system where flocculation is induced only by salt, the time scale involved in the rearrangement is estimated to be very short, possibly less than 1 second. Even for the polymerinduced flocculation, it is accepted that the rearrangement of the flocs takes place within a few seconds after adsorption, as demonstrated in the experiment using PEO as a flocculant [35]. However, a recent study by the authors [45] revealed that the order of a few hours is required for a polymeric layer adsorbed on a single colloidal particle to reach the state of equilibrium. Analysis on floc structures as well as factors acting on their determination in a system of wellproceeding levels of coagulation would be important to evaluate long-term behaviors of colloidal dispersions, such as the stability of the system and the breakage and/or regrow of the flocs. Electrophoresis, despite the ease of the measurement, remains unrevealed for the problem dealing with a particle carrying an ion-permeable polymeric layer around it. Theoretical work on the electrophoresis of socalled soft particles has been developed [46], though quantitative discussions are still under development including the charge distribution and internal polymer structures inside the polymeric layer and their correspondence to the experimental results. Furthermore, interior floc structures and the presence of polymeric interparticle matrix markedly influence the fluid flow within the flocs. This is regarded as a great importance for the electrophoretic transport, settling behavior of flocs, and biochemical interactions such as the exchange of substrates among microbial cells within a colony, and we expect further investigations.

\section{Acknowledgments}

The authors express their thanks to Dr. Kenji Aoki, Dr. Yasuyuki Kusaka, and Dr. Tomonori Fukasawa for their frank discussions. This work is partly funded by Grants-in-Aid for scientific research (222408025) from JSPS.

\section{References}

[1] Y. Adachi and S. Iwata, Eds., "Tsuchi no koroido genshou," Gakkai-Shuppan Sentar, pp. 1-451, 2003 (Japanese).

[2] E. P. Odum, Basic Ecology, CBS College Publishing, 1988.

[3] K. A. Hunter and P. S. Liss, "The surface charge of suspended particles in estuarine and coastal waters," Nature, vol. 282, no. 5741, pp. 823-825, 1979.

[4] L. M. Mosley, K. A. Hunter, and W. A. Ducker, "Forces between colloid particles in natural waters," Environmental Science and Technology, vol. 37, no. 15, pp. 3303-3308, 2003.

[5] K. Aoki and Y. Adachi, "Flocculation mechanisms of colloidal particles with polyelectrolytes bridging vs. Charge neutralization," Transactions of The Japanese Society of Irrigation, Drainage and Rural Engineering, vol. 245, pp. 65-71, 2006.

[6] M. J. Vold, "A numerical approach to the problem of sediment volume," Journal of Colloid Science, vol. 14, no. 2, pp. 168-174, 1959.
[7] M. J. Vold, "Computer simulation of floc formation in a colloidal suspension," Journal of Colloid Science, vol. 18, no. 7, pp. 684-695, 1963.

[8] D. N. Sutherland, "A theoretical model of floc structure," Journal of Colloid And Interface Science, vol. 25, no. 3, pp. 373-380, 1967.

[9] D. N. Sutherland and I. Goodarz-Nia, "Floc simulation: the effect of collision sequence," Chemical Engineering Science, vol. 26, no. 12, pp. 2071-2085, 1971.

[10] M. V. Smoluchowski, "Versuch einer mathematichen theorie der koagulations kinetik kollider losungen," Zeitschrift fur Physikalische Chemie, vol. 92, pp. 129-168, 1917.

[11] N. Tambo and Y. Watanabe, "Physical characteristics of flocsI. The floc density function and aluminium floc," Water Research, vol. 13, no. 5, pp. 409-419, 1979.

[12] Y. Adachi and S. Ooi, "Structure of a floc," in Proceedings of the World Congress III of Chemical Engineering, vol. 3, pp. 156$159,1986$.

[13] Y. Adachi and S. Ooi, "Geometrical structure of a floc," Journal of Colloid And Interface Science, vol. 135, no. 2, pp. 374-384, 1990.

[14] P. Meakin and R. Jullien, "The effects of restructuring on the geometry of clusters formed by diffusion-limited, ballistic, and reaction-limited cluster-cluster aggregation," The Journal of Chemical Physics, vol. 89, no. 1, pp. 246-250, 1988.

[15] Y. Adachi and Y. Tanaka, "Settling velocity of an aluminium-kaolinite floc," Water Research, vol. 31, no. 3, pp. 449-454, 1997.

[16] R. C. Sonntag and W. B. Russel, "Structure and breakup of flocs subjected to fluid stresses. I. Shear experiments," Journal of Colloid And Interface Science, vol. 113, no. 2, pp. 399-413, 1986.

[17] M. Kobayashi, "Breakup of fractal floes in a turbulent flow," Langmuir, vol. 15, no. 13, pp. 4351-4356, 1999.

[18] Y. Adachi, M. Kobayashi, and Y. Fukuhara, "Break-up strength of floes analyzed using orifice converging flow," Nihon Reoroji Gakkaishi, vol. 35, no. 2, pp. 69-72, 2007 (Japanese).

[19] M. Kobayashi, Y. Adachi, and S. Ooi, "On the steady shear viscosity of coagulated suspensions," Nihon Reoroji Gakkaishi, vol. 28, no. 3, pp. 143-144, 2000.

[20] M. Kobayashi, S. Ooi, and Y. Adachi, "On the yield stress of sheared coagulated suspensions," Annual Journal of Hydraulic Engineering, vol. 46, pp. 637-640, 2002.

[21] M. Y. Lin, H. M. Lindsay, D. A. Weitz, R. C. Ball, R. Klein, and P. Meakin, "Universality in colloid aggregation," Nature, vol. 339, no. 6223, pp. 360-362, 1989.

[22] T. Fukasawa and Y. Adachi, "Direct observation on the Brownian coagulation of PSL particles through optical microscope in the regime near critical coagulation concentration (CCC)," Journal of Colloid and Interface Science, vol. 344, no. 2, pp. 343347, 2010.

[23] Y. Kusaka, T. Fukasawa, and Y. Adachi, "Cluster-cluster aggregation simulation in a concentrated suspension," Journal of Colloid and Interface Science, vol. 363, no. 1, pp. 34-41, 2011.

[24] Y. Adachi, M. Kobayashi, and T. Yanagibashi, "Densification of small flocs formed in the mixing flow under the condition of rapid coagulation," Transactions of the Japanese Society of Irrigation, Drainage and Reclamation Engineering, vol. 233, pp. 49-56, 2004.

[25] K. Higashitani and T. Kubota, "Pelleting flocculation of colloidal latex particles," Powder Technology, vol. 51, no. 1, pp. 61-69, 1987.

[26] D. H. Napper, Polymeric Stabilization of Colloidal Dispersions, Academic Press, 1983. 
[27] R. A. Ruehrwein and D. W. Ward, "Mechanism and clay aggregation by polyelectrolytes," Soil Science, vol. 73, pp. 485-492, 1952.

[28] R. H. Smellie Jr. and V. K. La Mer, "Flocculation, subsidence and filtration of phosphate slimes. VI. A quantitative theory of filtration of flocculated suspensions," Journal of Colloid Science, vol. 13, no. 6, pp. 589-599, 1958.

[29] G. J. Fleer and J. Lyklema, "Polymer adsorption and its effect on the stability of hydrophobic colloids. II. The flocculation process as studied with the silver iodide-polyvinyl alcohol system," Journal of Colloid And Interface Science, vol. 46, no. 1, pp. $1-12,1974$.

[30] J. Gregory, "Rates of flocculation of latex particles by cationic polymers," Journal of Colloid And Interface Science, vol. 42, no. 2, pp. 448-456, 1973.

[31] K. Higashitani, T. Shibata, and H. Kage, "Formation of pellet flocs from kaoline suspension and their properties," Journal of Chemical Engineering of Japan, vol. 20, no. 2, pp. 152-157, 1987.

[32] Y. Adachi, M. A. Stuart, and R. Fokkink, "Kinetics of turbulent coagulation studied by means of end-over-end rotation," Journal of Colloid and Interface Science, vol. 165, no. 2, pp. 310-317, 1994.

[33] P. G. Saffman and J. S. Turner, "On the collision of drops in turbulent clouds," The Journal of Fluid Mechanics, vol. 1, pp. 16-30, 1956.

[34] T. G. M. van de Ven and S. G. Mason, "The microrheology of colloidal dispersions VII. Orthokinetic doublet formation of spheres," Colloid and Polymer Science, vol. 255, no. 5, pp. 468479, 1977.

[35] Y. Adachi, M. A. Stuart, and R. Fokkink, "Dynamic aspects of bridging flocculation studied using standardized mixing," Journal of Colloid And Interface Science, vol. 167, no. 2, pp. 346-351, 1994.

[36] Y. Adachi, "Dynamic aspects of coagulation and flocculation," Advances in Colloid and Interface Science, vol. 56, pp. 1-31, 1995.

[37] Y. Adachi and T. Matsumoto, "Dynamics of initial stage flocculation of polystyrene latex spheres with polyelectrolytes," Colloids and Surfaces A, vol. 113, no. 3, pp. 229-236, 1996.

[38] T. Matsumoto and Y. Adachi, "Effect of ionic strength on the initial dynamics of flocculation of polystyrene latex with polyelectrolyte," Journal of Colloid and Interface Science, vol. 204, no. 2, pp. 328-335, 1998.

[39] K. Aoki, Koubunshi denkaishitsu niyoru koroid ryushi no gyoshukatei ni kansuru kenkyu, Ph.D. thesis, University of Tsukuba, 2007.

[40] K. Aoki and Y. Adachi, "Kinetics of polyelectrolyte adsorption onto polystyrene latex particle studied using electrophoresis: effects of molecular weight and ionic strength," Journal of Colloid and Interface Science, vol. 300, no. 1, pp. 69-77, 2006.

[41] Y. Adachi and K. Aoki, "Restructuring of small flocs of polystyrene latex with polyelectrolyte," Colloids and Surfaces A, vol. 342, no. 1-3, pp. 24-29, 2009.

[42] J. Wei, B. Gao, Q. Yue, Y. Wang, W. Li, and X. Zhu, "Comparison of coagulation behavior and floc structure characteristic of different polyferric-cationic polymer dual-coagulants in humic acid solution," Water Research, vol. 43, no. 3, pp. 724$732,2009$.

[43] T. Fukasawa and Y. Adachi, "Effect of floc structure on the rate of Brownian coagulation," Journal of Colloid and Interface Science, vol. 304, no. 1, pp. 115-118, 2006.
[44] D. Sato, M. Kobayashi, and Y. Adachi, "Effect of floc structure on the rate of shear coagulation," Journal of Colloid and Interface Science, vol. 272, no. 2, pp. 345-351, 2004.

[45] Y. Adachi, Y. Kusaka, and A. Kobayashi, "Transient behavior of adsorbing/adsorbed polyelectrolytes on the surface of colloidal particles studied by means of trajectory analysis of Brownian motion," Colloids and Surfaces A, vol. 376, pp. 9-13, 2011.

[46] H. Ohshima, Biophysical Chemistry of Biointerfaces, John Wiley \& Sons, 2010.

[47] Y. Adachi and T. Wada, "Initial stage dynamics of bridging flocculation of polystyrene latex spheres with polyethylene oxide," Journal of Colloid and Interface Science, vol. 229, no. 1, pp. 148-154, 2000. 

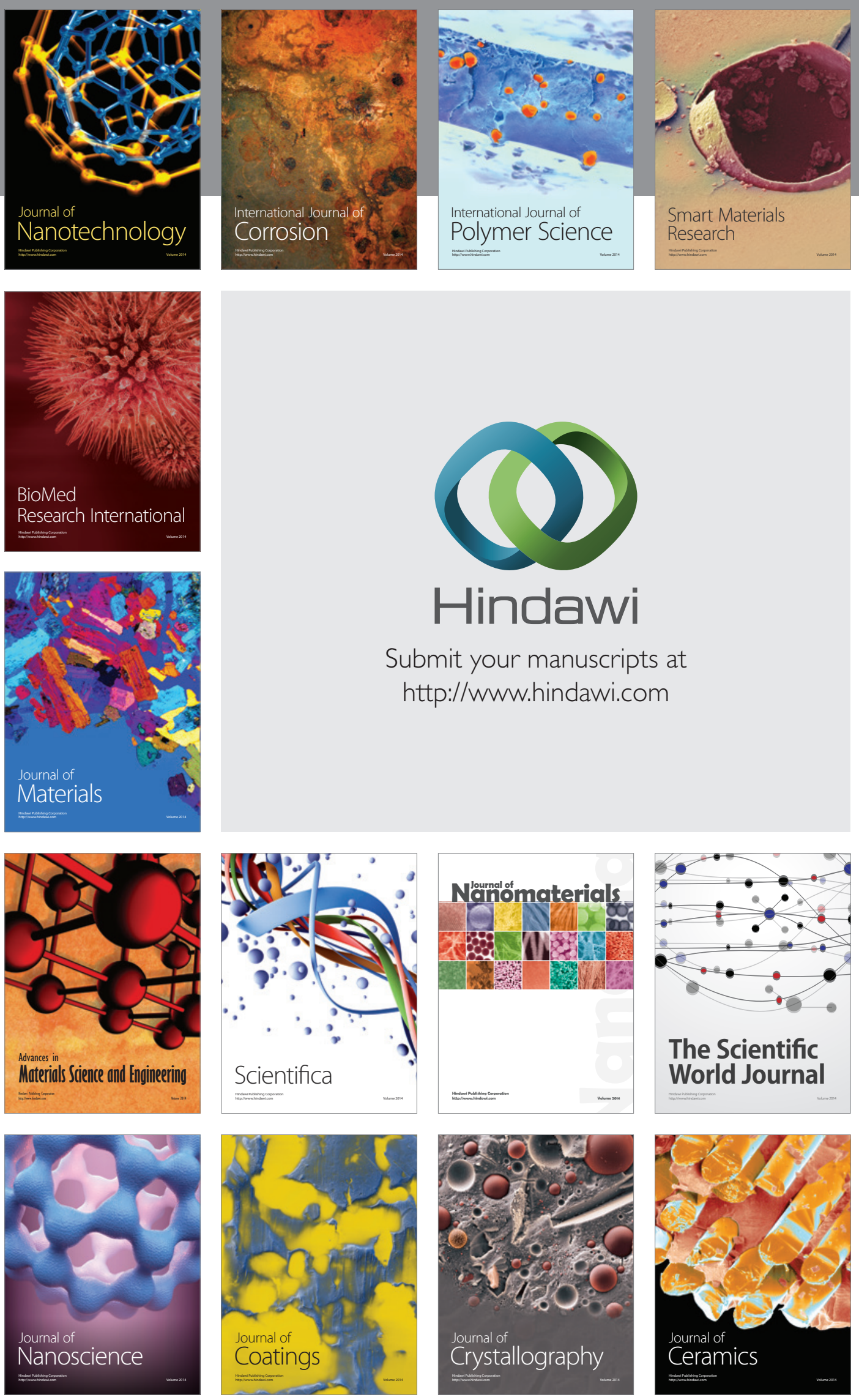

The Scientific World Journal

Submit your manuscripts at

http://www.hindawi.com

\section{World Journal}

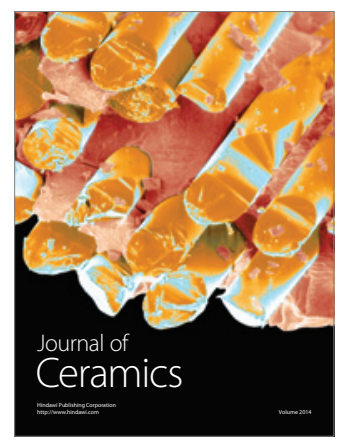

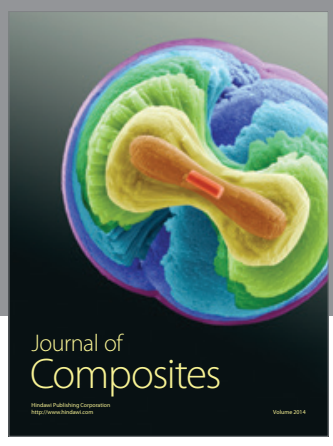
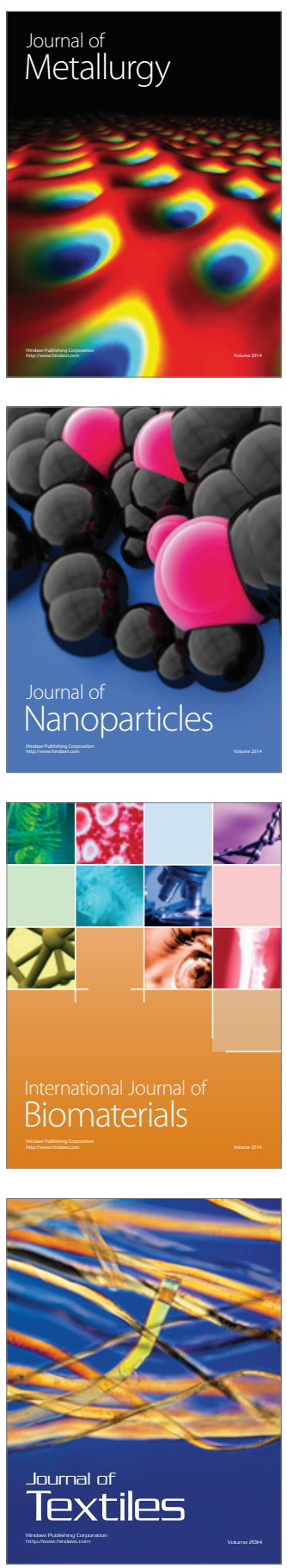